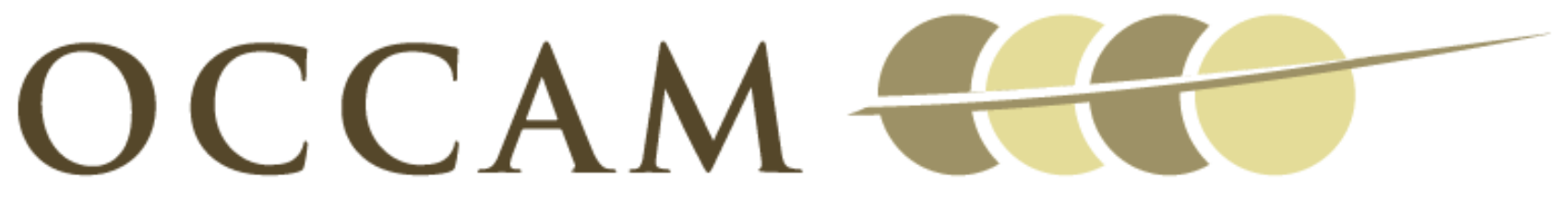

OXFORD CENTRE FOR COLLABORATIVE APPLIED MATHEMATICS

\author{
Report Number 11/25
}

Nonlinear waves in heterogeneous elastic rods via homogenization by

Manuel Quezada de Luna, Bojan Duričković and Alain Goriely

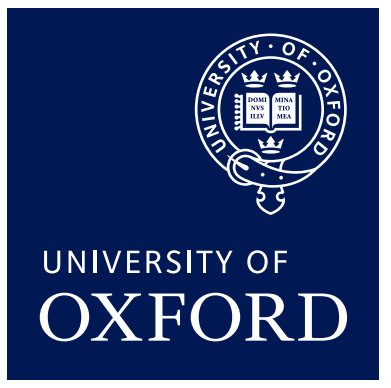

Oxford Centre for Collaborative Applied Mathematics Mathematical Institute 24 - 29 St Giles'

Oxford

OX1 3LB

England 



\title{
Nonlinear waves in heterogeneous elastic rods via homogenization
}

\author{
Manuel Quezada de Luna
}

Division of Mathematical and Computer Sciences \& Engineering King Abdullah University of Science and Technology, Saudi Arabia

\author{
Bojan Đuričković \\ Program in Applied Mathematics, \\ University of Arizona, Tucson, USA
}

\author{
Alain Goriely* \\ OCCAM, Mathematical Institute, \\ University of Oxford, Oxford, UK \\ goriely@maths.ox.ac.uk
}

February 7, 2011

\begin{abstract}
We consider the propagation of a planar loop on a heterogeneous elastic rod with a periodic microstructure consisting of two alternating homogeneous regions with different material properties. The analysis is carried out using a second-order homogenization theory based on a multiple scale asymptotic expansion.
\end{abstract}

\section{Introduction}

We consider an inextensible and unshearable elastic rod of circular cross-section and infinite length, that is straight in a stress-free state.

The static and dynamical solutions of such a system under fixed tension and with homogeneous material properties have been extensively studied and classified starting with the classical work of Euler and Kirchhoff, among others [1]. Owing to the remarkable analogy between the dynamical solutions of the spinning tops and the static solutions of this elastic rod, a solution connecting asymptotically the two straight states can be simply expressed in terms of

* Corresponding author 
the curvature by a sech function (this solution corresponds to the homoclinic solution connecting the upward pendulum to itself). As planar traveling-wavereduced equations are formally equivalent to static ones [2], this loop-like solution can also travel along the rod with constant velocity and represent the propagation of localized flexural waves.

A classical problem in the theory of homogenization is ti to consider longitudinal waves in a heterogeneous elastic medium with a periodic material microstructure of two alternating homogeneous materials. For the case of smallamplitude linear elastic wave, this problem has been analyzed using a homogenization technique [3], based on a multiple scale expansion, introducing a fast length variable on the scale of the microstructure. The leading order balance yields the effective homogeneous material properties, which is a crude approximation considering that it does not exhibit the dispersive behavior characteristic of the heterogeneous material, brought about by successive reflections on material interfaces. Dispersion is then captured by higher order corrections.

The goal of this paper is to investigate the effect of such heterogeneities in localized flexural waves on a straight elastic rod.

\section{Governing equations}

Geometrically, the rod is characterized by a curve called the centerline, and parametrized by the arc length $s$. We assume that the rod is inextensible, that is the parametrization $r(s)$ of the centerline is arc-length-preserving for all time, and unshearable, i.e. the cross-sections remain normal to the centerline tangent. Moreover, we assume that the rod is confined to the $x-y$ plane and ignore the possible effect of self-contact. Let $(x, y)$ be the coordinates of a point of the rod centerline, $(F, G)$ the coordinates of the force acting at that point, and $\Phi$ the angle the tangent vector at $(x, y)$ makes with the $x$-axis. The dynamics of the rod is then governed by the following system of equations (cf. e.g. [4]):

$$
\begin{aligned}
\rho A x_{t t} & =F_{s}, \\
\rho A y_{t t} & =G_{s}, \\
\rho I \Phi_{t t} & =\left(E I \Phi_{s}\right)_{s}+G \cos \Phi-F \sin \Phi, \\
x_{s} & =\cos \Phi, \\
y_{s} & =\sin \Phi,
\end{aligned}
$$

where $A$ and $I$ are the cross-section area and second moment of area, $\rho$ is the (mass) density, and $E$ is the Young modulus. We eliminate $x$ and $y$ from the first two equations by differentiating them with respect to $s$ and using the last two equations differentiated twice with respect to $t$. Thus we obtain the following 
system for $(F, G, \Phi)$ :

$$
\begin{aligned}
A(\cos \Phi)_{t t} & =\left(\frac{F_{s}}{\rho}\right)_{s}, \\
A(\sin \Phi)_{t t} & =\left(\frac{G_{s}}{\rho}\right)_{s}, \\
\rho I \Phi_{t t} & =\left(E I \Phi_{s}\right)_{s}+G \cos \Phi-F \sin \Phi .
\end{aligned}
$$

We assume that the rod is uniform with a circular cross-section of radius $R$, hence

$$
A=\pi R^{2}, \quad I=\frac{\pi R^{4}}{4} .
$$

The scaling:

$$
[s]=\frac{R}{2}, \quad[t]=1 s, \quad[F]=[G]=\pi R^{2}[E], \quad[E]=\frac{R^{2}}{4} \frac{[\rho]}{[t]^{2}},
$$

yields the following non-dimensionalized system (all variables are now dimensionless, but are denoted by the same symbol as their dimensional counterparts):

$$
\begin{aligned}
(\cos \Phi)_{t t} & =\left(\frac{F_{s}}{\rho}\right)_{s}, \\
(\sin \Phi)_{t t} & =\left(\frac{G_{s}}{\rho}\right)_{s}, \\
\rho \Phi_{t t} & =\left(E \Phi_{s}\right)_{s}+G \cos \Phi-F \sin \Phi .
\end{aligned}
$$

\section{Multiple scales asymptotic expansion}

We consider a rod with variable material properties on a small scale, so that regions of two different constant properties alternate periodically (see Figure 1). We denote the length of the unit cell by $\epsilon$. This cell is composed of two subdomains with lengths $\alpha \epsilon$ and $(1-\alpha) \epsilon$, densities $\rho_{a}$ and $\rho_{b}$, and Young moduli $E_{a}$ and $E_{b}$, respectively.

Assuming that the solution to the system (5) is essentially constant over a unit cell, i.e. that $\epsilon$ is a small parameter with respect to a characteristic length of the solution, we introduce a fast length scale $\hat{s}$ :

$$
\hat{s}=\frac{s}{\epsilon},
$$

and proceed with a standard multiple scale analysis (see e.g. [5]). The material 


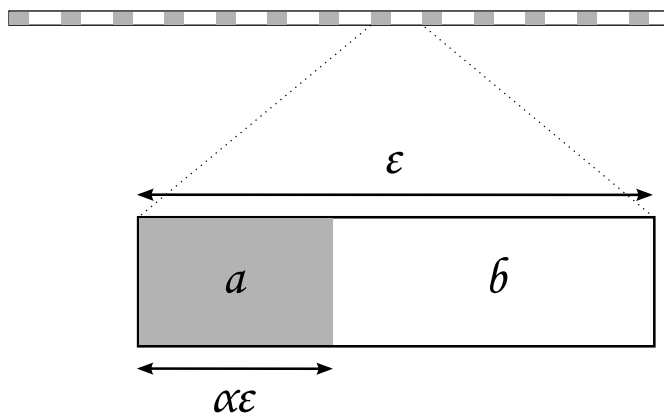

Figure 1: Heterogeneous rod microstructure

parameters are functions of the fast arc length $\hat{s}$ only,

$$
\begin{gathered}
\rho \equiv \rho(\hat{s}):=\left\{\begin{array}{ll}
\rho_{a}, & \hat{s} \in[0, \alpha) \\
\rho_{b}, & \hat{s} \in[\alpha, 1)
\end{array},\right. \\
E \equiv E(\hat{s}):=\left\{\begin{array}{ll}
E_{a}, & \hat{s} \in[0, \alpha) \\
E_{b}, & \hat{s} \in[\alpha, 1)
\end{array} .\right.
\end{gathered}
$$

The periodic structure of the rod induces periodicity in terms of the fast arc length variable $\hat{s}$ with period 1 (size of the unit cell in terms of $\hat{s}$ ) in the dependent variables in the system,

$$
F \equiv F(s, \hat{s}, t), \quad G \equiv G(s, \hat{s}, t), \quad \Phi \equiv \Phi(s, \hat{s}, t) .
$$

With the addition of the fast arc length variable, the spatial differential operator needs to be modified:

$$
\partial_{s} \mapsto \partial_{s}+\frac{1}{\epsilon} \partial_{\hat{s}}
$$

and the system is:

$$
\begin{aligned}
(\cos \Phi)_{t t} & =\left(\frac{F_{s}+\frac{1}{\epsilon} F_{\hat{s}}}{\rho}\right)_{s}+\frac{1}{\epsilon}\left(\frac{F_{s}+\frac{1}{\epsilon} F_{\hat{s}}}{\rho}\right)_{\hat{s}} \\
(\sin \Phi)_{t t} & =\left(\frac{G_{s}+\frac{1}{\epsilon} G_{\hat{s}}}{\rho}\right)_{s}+\frac{1}{\epsilon}\left(\frac{G_{s}+\frac{1}{\epsilon} G_{\hat{s}}}{\rho}\right)_{\hat{s}} \\
\rho \Phi_{t t} & =\left(E\left(\Phi_{s}+\frac{1}{\epsilon} \Phi_{\hat{s}}\right)\right)_{s}+\frac{1}{\epsilon}\left(E\left(\Phi_{s}+\frac{1}{\epsilon} \Phi_{\hat{s}}\right)\right)_{\hat{s}}+G \cos \Phi-F \sin \Phi
\end{aligned}
$$

We formally expand the variables in $\epsilon$,

$$
F=\sum_{i=0}^{\infty} \epsilon^{i} F_{i}(s, \hat{s}, t), \quad G=\sum_{i=0}^{\infty} \epsilon^{i} G_{i}(s, \hat{s}, t), \quad \Phi=\sum_{i=0}^{\infty} \epsilon^{i} \Phi_{i}(s, \hat{s}, t)
$$


and expand the trigonometric functions on the left-hand sides about $\Phi_{0}$, e.g.:

$$
\cos \Phi=\cos \Phi_{0}+\sum_{i=1}^{\infty} \frac{\cos ^{(i)} \Phi_{0}}{i !}\left(\sum_{j=1}^{\infty} \epsilon^{j} \Phi_{j}\right)^{i} .
$$

\section{1 $O\left(\epsilon^{-2}\right)$ system}

Collecting terms in the system (10) expanded via (11) and (12), the lowest order $O\left(\epsilon^{-2}\right)$ yields the following system:

$$
\begin{aligned}
& \left(\frac{F_{0, \hat{s}}}{\rho}\right)_{\hat{s}}=0, \\
& \left(\frac{G_{0, \hat{s}}}{\rho}\right)_{\hat{s}}=0, \\
& \left(E \Phi_{0, \hat{s}}\right)_{\hat{s}}=0 .
\end{aligned}
$$

Multiplying (13a) by $F_{0}$ and integrating by parts with respect to $\hat{s}$,

$$
\left.F_{0} \frac{F_{0, \hat{s}}}{\rho}\right|_{0} ^{1}-\int_{0}^{1} \frac{F_{0, \hat{s}}^{2}}{\rho} \mathrm{d} \hat{s}=0,
$$

the first term vanishes by periodicity of $F_{0}$, and, as expected, we conclude that $F_{0, \hat{s}} \equiv 0$, i.e. that $F_{0}$ is a function of $s$ and $t$ only. Equations (13b) and (13c) yield analogous results for $G_{0}$ and $\Phi_{0}$, thus

$$
F_{0} \equiv f_{0}(s, t), \quad G_{0} \equiv g_{0}(s, t), \quad \Phi_{0} \equiv \phi_{0}(s, t) .
$$

We consistently denote variables that do not explicitly depend on the fast arc length $\hat{s}$ with lowercase letters, and reserve uppercase letters for variables that depend on the rod microstructure.

\section{$3.2 O\left(\epsilon^{-1}\right)$ system}

The next order of $\epsilon$ in the system (10) expanded via (11) is $O\left(\epsilon^{-1}\right)$ :

$$
\begin{gathered}
\frac{F_{0, s \hat{s}}}{\rho}+\left(\frac{F_{0, s}+F_{1, \hat{s}}}{\rho}\right)_{\hat{s}}=0, \\
\frac{G_{0, s \hat{s}}}{\rho}+\left(\frac{G_{0, s}+G_{1, \hat{s}}}{\rho}\right)_{\hat{s}}=0, \\
E \Phi_{0, s \hat{s}}+\left(E \Phi_{0, s}+E \Phi_{1, \hat{s}}\right)_{\hat{s}}=0 .
\end{gathered}
$$

We solve equation (16a) for $F_{1}$ using the following ansatz (cf. $[3,6]$ ):

$$
F_{1}(s, \hat{s}, t)=f_{1}(s, t)+K(\hat{s}) f_{0, s}(s, t),
$$


where this decomposition is made unique by imposing the following normalization condition

$$
\left\langle F_{1}\right\rangle=f_{1}(s, t) \Longrightarrow\langle K\rangle=0,
$$

where the operator $\langle\cdot\rangle$ averages over the unit cell:

$$
\langle\cdot\rangle: \varphi \mapsto \int_{0}^{1} \varphi(x) \mathrm{d} x .
$$

Equation (16a) then implies the following ODE for $K$ :

$$
\left(\frac{1+K_{\hat{s}}}{\rho}\right)_{\hat{s}}=0 .
$$

Recall that $\rho$ is a piecewise-constant function (cf. (7a)). Integrating (20) over each subdomain, we obtain affine functions that we denote $K_{a}$ and $K_{b}$, respectively. The four integration constants (two on each subdomain) are found from the following conditions:

(a) continuity (in terms of $\hat{s}$ ) of $F_{1}$,

(b) periodicity (in terms of $\hat{s}$ ) of $F_{1}$,

(c) the normalization condition (18),

(d) continuity of the parenthesized expression in (20), which we term the validation condition.

Conditions (a) and (b) imply continuity and periodicity of $K$, respectively, yielding $K_{a}(\alpha)=K_{b}(\alpha)$ and $K_{a}(0)=K_{b}(1)$. Note that the validation condition (d) implies differentiability, since the derivative of the expression vanishes on both intervals, hence on both sides of the point $\hat{s}=\alpha$. The four conditions yield the following solution

$$
K(\hat{s})=\left\{\begin{array}{ll}
K_{a}(\hat{s}):=\frac{(1-\alpha)\left(\rho_{a}-\rho_{b}\right)}{\alpha \rho_{a}+(1-\alpha) \rho_{b}}\left(\hat{s}-\frac{\alpha}{2}\right), & \hat{s} \in[0, \alpha) \\
K_{b}(\hat{s}):=\frac{\alpha\left(\rho_{a}-\rho_{b}\right)}{\alpha \rho_{a}+(1-\alpha) \rho_{b}}\left(\frac{1+\alpha}{2}-\hat{s}\right), & \hat{s} \in[\alpha, 1)
\end{array} .\right.
$$

By symmetry, equation (16b) yields

$$
G_{1}(s, \hat{s}, t)=g_{1}(s, t)+K(\hat{s}) g_{0, s}(s, t),
$$

where $K$ is also given by $(21)$, and $g_{1}(s, t) \equiv\left\langle G_{1}\right\rangle$.

The solution to (16c) is entirely analogous. The ansatz

$$
\Phi_{1}(s, \hat{s}, t)=\phi_{1}(s, t)+L(\hat{s}) \phi_{0, s}(s, t),
$$

with the normalization condition

$$
\left\langle\Phi_{1}\right\rangle=\phi_{1}(s, t) \Longrightarrow\langle L\rangle=0,
$$


yields the following ODE for $L$ :

$$
\left(E\left(1+L_{\hat{s}}\right)\right)_{\hat{s}}=0
$$

with the following solution:

$$
L(\hat{s})=\left\{\begin{array}{ll}
L_{a}(\hat{s}):=\frac{(1-\alpha)\left(E_{b}-E_{a}\right)}{\left(1-\alpha E_{a}+\alpha E_{b}\right.}\left(\hat{s}-\frac{\alpha}{2}\right), & \hat{s} \in[0, \alpha) \\
L_{b}(\hat{s}):=\frac{\alpha\left(E_{b}-E_{a}\right)}{(1-\alpha) E_{a}+\alpha E_{b}}\left(\frac{1+\alpha}{2}-\hat{s}\right), & \hat{s} \in[\alpha, 1)
\end{array} .\right.
$$

For future reference, we note that the equations (20) and (25) along with the validation conditions imply that the differentiated expressions are constant over the unit cell. We can evaluate these constants using the solutions (21) and (26) for $K$ and $L$, respectively:

$$
\begin{gathered}
\frac{1+K_{\hat{s}}}{\rho} \equiv \frac{1}{\alpha \rho_{a}+(1-\alpha) \rho_{b}}=\langle\rho\rangle^{-1}=: \rho_{h}^{-1}, \\
E\left(1+L_{\hat{s}}\right) \equiv \frac{E_{a} E_{b}}{(1-\alpha) E_{a}+\alpha E_{b}}=\left\langle E^{-1}\right\rangle^{-1}=: E_{h} .
\end{gathered}
$$

\section{$3.3 \quad O\left(\epsilon^{0}\right)$ system}

Next, we consider the system of order $O\left(\epsilon^{0}\right)$ :

$$
\begin{aligned}
\left(\cos \Phi_{0}\right)_{t t} & =\left(\frac{F_{0, s}+F_{1, \hat{s}}}{\rho}\right)_{s}+\left(\frac{F_{1, s}+F_{2, \hat{s}}}{\rho}\right)_{\hat{s}} \\
\left(\sin \Phi_{0}\right)_{t t} & =\left(\frac{G_{0, s}+G_{1, \hat{s}}}{\rho}\right)_{s}+\left(\frac{G_{1, s}+G_{2, \hat{s}}}{\rho}\right)_{\hat{s}} \\
\rho \Phi_{0, t t} & =\left(E\left(\Phi_{0, s}+\Phi_{1, \hat{s}}\right)\right)_{s}+\left(E\left(\Phi_{1, s}+\Phi_{2, \hat{s}}\right)\right)_{\hat{s}}+G_{0} \cos \Phi_{0}-F_{0} \sin \Phi_{0}
\end{aligned}
$$

Using the ansatz (17), (22), (23) for $F_{1}, G_{1}, \Phi_{1}$, as well as identities (27), the system (28) becomes:

$$
\begin{aligned}
\left(\cos \phi_{0}\right)_{t t} & =\frac{f_{0, s s}}{\rho_{h}}+\left(\frac{f_{1, s}+K f_{0, s s}+F_{2, \hat{s}}}{\rho}\right)_{\hat{s}} \\
\left(\sin \phi_{0}\right)_{t t} & =\frac{g_{0, s s}}{\rho_{h}}+\left(\frac{g_{1, s}+K g_{0, s s}+G_{2, \hat{s}}}{\rho}\right)_{\hat{s}} \\
\rho \phi_{0, t t} & =E_{h} \phi_{0, s s}+\left(E\left(\phi_{1, s}+L \phi_{0, s s}+\Phi_{2, \hat{s}}\right)\right)_{\hat{s}}+g_{0} \cos \phi_{0}-f_{0} \sin \phi_{0}
\end{aligned}
$$

\subsubsection{Averaged $O\left(\epsilon^{0}\right)$ system}

We apply the averaging operator $\langle\cdot\rangle(19)$ on the system $(29)$. We note that $\left\langle\varphi_{\hat{s}}\right\rangle \equiv 0$ for any function $\varphi$ periodic on a unit cell, hence the second terms on the right hand sides of (29) all vanish when averaged. The $O\left(\epsilon^{0}\right)$ balance is thus 


$$
\begin{aligned}
\left(\cos \phi_{0}\right)_{t t} & =\frac{f_{0, s s}}{\rho_{h}} \\
\left(\sin \phi_{0}\right)_{t t} & =\frac{g_{0, s s}}{\rho_{h}} \\
\rho_{h} \phi_{0, t t} & =E_{h} \phi_{0, s s}+g_{0} \cos \phi_{0}-f_{0} \sin \phi_{0}
\end{aligned}
$$

This is a system describing the homogenized behavior of the heterogeneous rod in the leading order approximation. It has the form of a system of equations describing a homogeneous rod (cf. (5)), where the constant material properties are the bulk density $\rho_{h}$, and $E_{h}$, which is one-half of the harmonic average of the Young modulus (cf. (27)). Up to now, the analysis of the system was general. We now focus on the localized flexural waves in order to understand the effect on the microstructure in their characteristics. To do so, we solve the system (30) by a traveling wave reduction:

$$
\xi=s-c t, \quad \partial_{t} \mapsto-c \partial_{\xi}, \quad \partial_{s} \mapsto \partial_{\xi}
$$

The reduced system is

$$
\begin{aligned}
c^{2} \rho_{h}\left(\cos \phi_{0}\right)_{\xi \xi} & =f_{0, \xi \xi} \\
c^{2} \rho_{h}\left(\sin \phi_{0}\right)_{\xi \xi} & =g_{0, \xi \xi} \\
\left(c^{2} \rho_{h}-E_{h}\right) \phi_{0, \xi \xi} & =g_{0} \cos \phi_{0}-f_{0} \sin \phi_{0}
\end{aligned}
$$

The solutions we are looking for are loops on a straight rod with a tension $T$ applied at infinity. We thus impose the following boundary conditions at infinity for the force,

$$
\lim _{\xi \rightarrow \pm \infty} f_{0}=T, \quad \lim _{\xi \rightarrow \pm \infty} g_{0}=0,
$$

while the boundary conditions for the angle for a single loop are

$$
\lim _{\xi \rightarrow-\infty} \phi_{0}=0, \lim _{\xi \rightarrow+\infty} \phi_{0}=2 \pi .
$$

Integrating twice equations (32a) and (32b) subject to the above boundary conditions, we have

$$
\begin{aligned}
& f_{0}=c^{2} \rho_{h}\left(\cos \phi_{0}-1\right)+T, \\
& g_{0}=c^{2} \rho_{h} \sin \phi_{0} .
\end{aligned}
$$

Now we can eliminate $f_{0}$ and $g_{0}$ from $(32 \mathrm{c})$ :

$$
\phi_{0, \xi \xi}=\frac{1}{\ell^{2}} \sin \phi_{0},
$$

where

$$
\ell^{2}:=\frac{E_{h}-c^{2} \rho_{h}}{T-c^{2} \rho_{h}} .
$$


As expected from the Kirchhoff analogy, Equation (36) is the pendulum equation where the tangent angle plays the role of the angle the pendulum makes with the vertical and arc length corresponds to time [1]. The boundary conditions (34) correspond to a homoclinic orbit, with $\phi_{0}=0 \bmod 2 \pi$ as the homoclinic point. We therefore conclude that $\ell^{2}>0$ (a negative value of $\ell^{2}$ would have $\phi_{0}=\pi$ for a homoclinic point), which implies the following condition on the wave speed:

$$
\begin{gathered}
c^{2} \in \mathbb{R}^{+} \backslash\left[c_{1}{ }^{2}, c_{2}{ }^{2}\right], \\
c_{1}{ }^{2}:=\min \left(\frac{E_{h}}{\rho_{h}}, \frac{T}{\rho_{h}}\right), \quad c_{2}{ }^{2}:=\max \left(\frac{E_{h}}{\rho_{h}}, \frac{T}{\rho_{h}}\right) .
\end{gathered}
$$

Note that $\ell \rightarrow 0$ when the wave speed approaches $c_{0}:=\sqrt{E_{h} / \rho_{h}}$ (speed of sound in a homogeneous material with Young modulus $E_{h}$ and density $\rho_{h}$ ), and $\ell \rightarrow \infty$ for $c^{2} \rightarrow T / \rho_{h}$. With zero tension, the parameter $\ell \equiv \sqrt{1-c_{0}^{2} / c^{2}}$ is an increasing function of the wave speed $c$, and (38) yields a lower bound $c_{0}$ for the wave speed. Therefore, we have $0<\ell<1$, where $\ell \rightarrow 0$ for $c \rightarrow c_{0}$, and $\ell \rightarrow 1$ for $c \rightarrow \infty$. The solution to (36) with boundary conditions (34) is the well known homoclinic orbit of the pendulum (cf. [1]),

$$
\phi_{0}(\xi)=4 \arctan \mathrm{e}^{\frac{\xi-\xi_{0}}{\ell}},
$$

where $\xi_{0}$ is an integration constant that corresponds to the position of the midpoint of the loop. The parameter $\ell$ can now be identified as the characteristic size of the loop. The solution (39) is shown in Figure 2. The shape of the planar rod corresponding to this tangent angle is a single loop that straightens out exponentially on the two ends, and is shown in Figure 5.

\subsubsection{Ansatz for $F_{2}, G_{2}, \Phi_{2}$}

We now go back to the $O\left(\epsilon^{0}\right)$ system (29). The averaged balance (30) implies

$$
\begin{aligned}
\left(\frac{f_{1, s}+K f_{0, s s}+F_{2, \hat{s}}}{\rho}\right)_{\hat{s}} & =0 \\
\left(\frac{g_{1, s}+K g_{0, s s}+G_{2, \hat{s}}}{\rho}\right)_{\hat{s}} & =0 \\
\left(E\left(\phi_{1, s}+L \phi_{0, s s}+\Phi_{2, \hat{s}}\right)\right)_{\hat{s}} & =\left(\rho-\rho_{h}\right) \phi_{0, t t}
\end{aligned}
$$

Following the ansatz in (17), we decompose $F_{2}, G_{2}$, and $\Phi_{2}$ as follows

$$
\begin{aligned}
& F_{2}(s, \hat{s}, t)=f_{2}(s, t)+K(\hat{s}) f_{1, s}(s, t)+M(\hat{s}) f_{0, s s}(s, t), \\
& G_{2}(s, \hat{s}, t)=g_{2}(s, t)+K(\hat{s}) g_{1, s}(s, t)+M(\hat{s}) g_{0, s s}(s, t), \\
& \Phi_{2}(s, \hat{s}, t)=\phi_{2}(s, t)+L(\hat{s}) \phi_{1, s}(s, t)+N(\hat{s}) \phi_{0, s s}(s, t),
\end{aligned}
$$

subject to the following normalization

$$
\begin{aligned}
& \langle M\rangle=0, \\
& \langle N\rangle=0,
\end{aligned}
$$


which is equivalent to

$$
\left\langle F_{2}\right\rangle=f_{2}(s, t), \quad\left\langle G_{2}\right\rangle=g_{2}(s, t), \quad\left\langle\Phi_{2}\right\rangle=\phi_{2}(s, t) .
$$

Equation (40a) becomes:

$$
\left(\frac{1+K_{\hat{s}}}{\rho}\right)_{\hat{s}} f_{1, s}(s, t)+\left(\frac{K+M_{\hat{s}}}{\rho}\right)_{\hat{s}} f_{0, s s}(s, t)=0
$$

The first term vanishes by (20), leaving

$$
\left(\frac{K+M_{\hat{s}}}{\rho}\right)_{\hat{s}}=0 .
$$

We solve this equation for $M$ analogously to (20), by integrating over the two subdomains separately. Recall that on each subdomain $\rho$ is constant (cf. (7a)), while $K$ is an affine function (cf. (21)). We thus obtain two quadratic functions $M_{a}, M_{b}$, where the integration constants are obtained from:

(a) continuity (in terms of $\hat{s}$ ) of $F_{2}$,

(b) periodicity (in terms of $\hat{s}$ ) of $F_{2}$,

(c) the normalization condition (42a)

(d) the validation condition: continuity of the parenthesized expression in (45).

The solution for $M$ is

$$
M(\hat{s})= \begin{cases}M_{a}(\hat{s}):=-\frac{(1-\alpha)\left(\rho_{a}-\rho_{b}\right)}{2 \rho_{h}}\left(\hat{s}^{2}-\alpha \hat{s}+\frac{\alpha(2 \alpha-1)}{6}\right), & \hat{s} \in[0, \alpha) \\ M_{b}(\hat{s}):=\frac{\alpha\left(\rho_{a}-\rho_{b}\right)}{2 \rho_{h}}\left(\hat{s}^{2}-(1+\alpha) \hat{s}+\frac{2 \alpha^{2}+3 \alpha+1}{6}\right), & \hat{s} \in[\alpha, 1)\end{cases}
$$

Similarly, we find $N$ from (40c),

$$
\left(\rho-\rho_{h}\right) \phi_{0, t t}=\left(E\left(L+N_{\hat{s}}\right)\right)_{\hat{s}} \phi_{0, s s} .
$$

Noting that $\phi_{0}$ satisfies the wave equation with speed $c$, this leads to

$$
\left(E\left(L+N_{\hat{s}}\right)\right)_{\hat{s}}=c^{2}\left(\rho-\rho_{h}\right) .
$$

Integrating the equation on the two subdomains where $E$ and $\rho$ are constants, as $L$ is affine function on each, we obtain two quadratic functions $N_{a}$ and $N_{b}$. The constants of integration are obtained by imposing the same four conditions as above. We note, however, that the validation condition, i.e. the continuity of $\left(E\left(L+N_{\hat{s}}\right)\right)$ here does not implies differentiability, as the right-hand side of (48) has a jump at the material interface $\hat{s}=\alpha$. Therefore, the solution obtained for $N$ is a weak solution.

$$
N(\hat{s})=\left\{\begin{array}{ll}
N_{a}(\hat{s}):=n_{a 2} \hat{s}^{2}+n_{a 1} \hat{s}+n_{a 0}, & \hat{s} \in[0, \alpha) \\
N_{b}(\hat{s}):=n_{b 2} \hat{s}^{2}+n_{b 1} \hat{s}+n_{b 0}, & \hat{s} \in[\alpha, 1)
\end{array},\right.
$$

where the $n$ 's are constants that depend on the parameters $\alpha, \rho_{a}, \rho_{b}, E_{a}, E_{b}$, and the wave speed $c$. (Henceforth, we omit the explicit expressions for the coefficients as they become rather cumbersome at this point.) 


\section{$3.4 O(\epsilon)$ system}

The next order system we consider is $O(\epsilon)$ :

$$
\begin{aligned}
-\left(\Phi_{1} \sin \Phi_{0}\right)_{t t}= & \left(\frac{F_{1, s}+F_{2, \hat{s}}}{\rho}\right)_{s}+\left(\frac{F_{2, s}+F_{3, \hat{s}}}{\rho}\right)_{\hat{s}} \\
\left(\Phi_{1} \cos \Phi_{0}\right)_{t t}= & \left(\frac{G_{1, s}+G_{2, \hat{s}}}{\rho}\right)_{s}+\left(\frac{G_{2, s}+G_{3, \hat{s}}}{\rho}\right)_{\hat{s}} \\
\rho \Phi_{1, t t}= & \left(E\left(\Phi_{1, s}+\Phi_{2, \hat{s}}\right)\right)_{s}+\left(E\left(\Phi_{2, s}+\Phi_{3, \hat{s}}\right)\right)_{\hat{s}}+G_{1} \cos \Phi_{0} \\
& -G_{0} \Phi_{1} \sin \Phi_{0}-F_{1} \sin \Phi_{0}-F_{0} \Phi_{1} \cos \Phi_{0}
\end{aligned}
$$

Applying the ansatz for $\left(F_{1}, G_{1}, \Phi_{1}\right)$ and $\left(F_{2}, G_{2}, \Phi_{2}\right)$, as well as the identity (27a) and the following one obtained from (45) and the solutions for $K(21)$ and $M(46)$ :

$$
\frac{K+M_{\hat{s}}}{\rho} \equiv 0,
$$

the system (50) becomes:

$$
\begin{aligned}
-\left(\left(\phi_{1}+L \phi_{2, s}\right) \sin \phi_{0}\right)_{t t}= & \frac{f_{1, s s}}{\rho_{h}}+\left(\frac{f_{2, s}+K f_{1, s s}+M f_{0, s s s}+F_{3, \hat{s}}}{\rho}\right)_{\hat{s}} \\
\left(\left(\phi_{1}+L \phi_{2, s}\right) \cos \phi_{0}\right)_{t t}= & \frac{g_{1, s s}}{\rho_{h}}+\left(\frac{g_{2, s}+K g_{1, s s}+M g_{0, s s s}+G_{3, \hat{s}}}{\rho}\right)_{\hat{s}} \\
\rho\left(\phi_{1, t t}+L \phi_{0, s t t}\right)= & E_{h} \phi_{1, s s}+E\left(L+N_{\hat{s}}\right) \Phi_{0, s s s} \\
& +\left(E\left(\phi_{2, s}+L \phi_{1, s s}+N \phi_{0, s s s}+\Phi_{3, \hat{s}}\right)\right)_{\hat{s}} \\
& +\left(g_{1}+K g_{0, s}\right) \cos \phi_{0}-g_{0}\left(\phi_{1}+L \phi_{0, s}\right) \sin \phi_{0} \\
& -\left(f_{1}+K f_{0, s}\right) \sin \phi_{0}-f_{0}\left(\phi_{1}+L \phi_{0, s}\right) \cos \phi_{0}
\end{aligned}
$$

\subsubsection{Averaged $O(\epsilon)$ system}

Averaging the system (50) over the unit cell, and using the following identities:

$$
\begin{aligned}
\left\langle E\left(L+N_{\hat{s}}\right)\right\rangle & =0, \\
\langle\rho L\rangle & =0,
\end{aligned}
$$

we obtain the $O(\epsilon)$ balance:

$$
\begin{aligned}
-\rho_{h}\left(\phi_{1} \sin \phi_{0}\right)_{t t}= & f_{1, s s} \\
\rho_{h}\left(\phi_{1} \cos \phi_{0}\right)_{t t}= & g_{1, s s} \\
\rho_{h} \phi_{1, t t}= & E_{h} \phi_{1, s s}-\phi_{1}\left(g_{0} \sin \phi_{0}+f_{0} \cos \phi_{0}\right) \\
& +g_{1} \cos \phi_{0}-f_{1} \sin \phi_{0}
\end{aligned}
$$

As the zeroth-order solution $\left(f_{0}, g_{0}, \phi_{0}\right)$ is known (cf. (35a), (35b), (39)), this is a system of equations for $\left(f_{1}, g_{1}, \phi_{1}\right)$. Applying a traveling wave reduction, and 
integrating the first two equations with zero boundary conditions at infinity, we have

$$
\begin{aligned}
& f_{1}=-c^{2} \rho_{h} \phi_{1} \sin \phi_{0} \\
& g_{1}=c^{2} \rho_{h} \phi_{1} \cos \phi_{0}
\end{aligned}
$$

Eliminating $f_{1}, g_{1}$ from the reduced third equation yields

$$
\phi_{1, \xi \xi}=\frac{1}{\ell^{2}} \phi_{1} \cos \phi_{0},
$$

where $\ell$ is given by (37). The solution of (56) satisfying the boundary conditions is given by

$$
\phi_{1}(\xi)=\phi_{0, \xi}(\xi)=\frac{2}{\ell} \operatorname{sech} \frac{\xi-\xi_{0}}{\ell},
$$

where $\phi_{0}$ is the zeroth-order solution (39). This is the first correction in the homogenized solution, one that captures (the leading order of) the difference with respect to the homogeneous system behavior.

\subsubsection{Ansatz for $F_{3}, G_{3}, \Phi_{3}$}

We now go back to the $O(\epsilon)$ system (50), with the following ansatz for $F_{3}, G_{3}, \Phi_{3}$ :

$$
\begin{aligned}
F_{3}(s, \hat{s}, t) & =f_{3}(s, t)+K(\hat{s}) f_{2, s}(s, t)+M(\hat{s}) f_{1, s s}(s, t)+P(\hat{s}) f_{0, s s s}(s, t) \\
G_{3}(s, \hat{s}, t) & =g_{3}(s, t)+K(\hat{s}) g_{2, s}(s, t)+M(\hat{s}) g_{1, s s}(s, t)+P(\hat{s}) g_{0, s s s}(s, t) \\
\Phi_{3}(s, \hat{s}, t) & =\phi_{3}(s, t)+L(\hat{s}) \phi_{2, s}(s, t)+N(\hat{s}) \phi_{1, s s}(s, t)+Q(\hat{s}) \phi_{0, s s s}(s, t),
\end{aligned}
$$

where $P$ and $Q$ satisfy the normalization conditions

$$
\langle P\rangle=0, \quad\langle Q\rangle=0,
$$

i.e.

$$
f_{3}(s, t)=\left\langle F_{3}\right\rangle, \quad g_{3}(s, t)=\left\langle G_{3}\right\rangle, \quad \phi_{3}(s, t)=\left\langle\Phi_{3}\right\rangle .
$$

Using the ansatz for $\left(F_{1}, G_{1}, \Phi_{1}\right)$ and $\left(F_{2}, G_{2}, \Phi_{2}\right)$, as well as the solution (57) and the identities (27), and (53), the system (50) becomes:

$$
\begin{aligned}
L\left(\cos \phi_{0}\right)_{s t t}= & \left(\frac{M+P_{\hat{s}}}{\rho}\right)_{\hat{s}} f_{0, s s s} \\
L\left(\sin \phi_{0}\right)_{s t t}= & \left(\frac{M+P_{\hat{s}}}{\rho}\right)_{\hat{s}} g_{0, s s s} \\
\rho L \phi_{0, s t t}= & {\left[E\left(L+N_{\hat{s}}\right)+\left(E\left(N+Q_{\hat{s}}\right)\right)_{\hat{s}}\right] \phi_{0, s s s} } \\
& \quad+c^{2}\langle\rho\rangle\left(K-L\left(1-\cos \phi_{0}\right)\right) \phi_{0, s}
\end{aligned}
$$

Transforming the left-hand side with the help of the averaged $O(1)$ system (30), each of the first two equations reduces to:

$$
\langle\rho\rangle^{-1} L=\left(\frac{M+P_{\hat{s}}}{\rho}\right)_{\hat{s}}
$$


As $L$ is an affine function, and $M$ is a quadratic one, the solution for $P$ is a cubic function each subdomain,

$$
P(\hat{s})= \begin{cases}P_{a}(\hat{s}):=p_{a 3} \hat{s}^{3}+p_{a 2} \hat{s}^{2}+p_{a 1} \hat{s}+p_{a 0}, & \hat{s} \in[0, \alpha) \\ P_{b}(\hat{s}):=p_{b 3} \hat{s}^{3}+p_{b 2} \hat{s}^{2}+p_{b 1} \hat{s}+p_{b 0}, & \hat{s} \in[\alpha, 1)\end{cases}
$$

where the coefficients $p$ are determined by the usual conditions: continuity, periodicity, normalization and validation, and are functions of the material parameters $E_{a}, E_{b}, \rho_{a}, \rho_{b}, \alpha$, and the wave speed $c$.

Since $\phi_{0}$ satisfies the wave equation with wave speed $c$, equation (61c) yields:

$$
\begin{aligned}
& {\left[c^{2} \rho L-E\left(L+N_{\hat{s}}\right)-\left(E\left(N+Q_{\hat{s}}\right)\right)_{\hat{s}}\right] \phi_{0, s s s}=} \\
& c^{2} \rho_{h}\left(K \cos 2 \phi_{0}-L\left(1-\cos \phi_{0}\right)\right) \phi_{0, s}
\end{aligned}
$$

This equation does not yield an ODE for $Q$ because of the extra term on the right-hand side. We therefore amend the ansatz (58c) for $\Phi_{3}$ so as to cancel this term out. The modified ansatz is

$$
\Phi_{3}(s, \hat{s}, t)=\phi_{3}+L \phi_{2, s}+N \phi_{1, s s}+Q \phi_{0, s s s}+H(s, \hat{s}, t),
$$

$H$ being a function such that

$$
\left(E H_{\hat{s}}\right)_{\hat{s}}=K \chi(s, t)+L \lambda(s, t)
$$

where

$$
\begin{aligned}
\chi(s, t) & :=c^{2} \rho_{h} \phi_{0, s} \cos 2 \phi_{0} \\
\lambda(s, t) & :=-c^{2} \rho_{h} \phi_{0, s}\left(1-\cos \phi_{0}\right)
\end{aligned}
$$

Moreover, we impose the following normalization condition on $H$ :

$$
\langle H\rangle=0,
$$

so that $\phi_{3}=\left\langle\Phi_{3}\right\rangle$ still holds, and we require $H$ to be continuous and periodic. Analogously to the validation conditions seen above, we require $E H_{\hat{s}}$ to be continuous as well. We obtain $H$ by integrating equation (66) twice over each of the two subdomains, and determine the integration constants from the aforementioned conditions on $H$. It is clear that the $\hat{s}$-dependence of $H$ is cubic. Note that integrating equation (66) with respect to $\hat{s}$ leaves $\chi$ and $\lambda$ intact, therefore the form of the solution for $H$ is

$$
H(s, \hat{s}, t)=H_{K}(\hat{s}) \chi(s, t)+H_{L}(\hat{s}) \lambda(s, t)
$$

The new ansatz (65) cancels out the last two terms in the right-hand side of (64), yielding the following ODE for $Q$ :

$$
\left(E\left(N+Q_{\hat{s}}\right)\right)_{\hat{s}}=\left(c^{2} \rho-E\right) L-E N_{\hat{s}}
$$


As previously, $Q$ is obtained by integrating twice over each of the two subdomains, and the integration constants are found the usual way. As $L$ is an affine function, and $N$ is quadratic, the resulting function $Q$ is a cubic on each subdomain:

$$
Q(\hat{s})= \begin{cases}Q_{a}(\hat{s}):=q_{a 3} \hat{s}^{3}+q_{a 2} \hat{s}^{2}+q_{a 1} \hat{s}+q_{a 0}, & \hat{s} \in[0, \alpha) \\ Q_{b}(\hat{s}):=q_{b 3} \hat{s}^{3}+q_{b 2} \hat{s}^{2}+q_{b 1} \hat{s}+q_{b 0}, & \hat{s} \in[\alpha, 1)\end{cases}
$$

\section{5 $O\left(\epsilon^{2}\right)$ system}

Collected terms of order $\epsilon^{2}$ yield the following system:

$$
\begin{gathered}
\left(-\Phi_{2} \sin \Phi_{0}-\frac{1}{2} \Phi_{1}^{2} \cos \Phi_{0}\right)_{t t}=\left(\frac{F_{2, s}+F_{3, \hat{s}}}{\rho}\right)_{s}+\left(\frac{F_{3, s}+F_{4, \hat{s}}}{\rho}\right)_{\hat{s}} \\
\left(\Phi_{2} \cos \Phi_{0}-\frac{1}{2} \Phi_{1}^{2} \sin \Phi_{0}\right)_{t t}=\left(\frac{G_{2, s}+G_{3, \hat{s}}}{\rho}\right)_{s}+\left(\frac{G_{3, s}+G_{4, \hat{s}}}{\rho}\right)_{\hat{s}} \\
\rho \Phi_{2, t t}=E\left(\Phi_{2, s}+\Phi_{3, \hat{s}}\right)_{s}+\left(E\left(\Phi_{3, s}+\Phi_{4, \hat{s}}\right)\right)_{\hat{s}} \\
+G_{0}\left(-\Phi_{2} \sin \Phi_{0}-\frac{1}{2} \Phi_{1}{ }^{2} \cos \Phi_{0}\right)-G_{1} \Phi_{1} \sin \Phi_{0}+G_{2} \cos \Phi_{0} \\
-F_{0}\left(\Phi_{2} \cos \Phi_{0}-\frac{1}{2} \Phi_{1}{ }^{2} \sin \Phi_{0}\right)-F_{1} \Phi_{1} \cos \Phi_{0}-F_{2} \sin \Phi_{0}
\end{gathered}
$$

\subsubsection{Averaged $O\left(\epsilon^{2}\right)$ system}

Using the ansatz expressions and the known identities, the averaged system (72) becomes

$$
\begin{aligned}
-\left(\phi_{2} \sin \phi_{0}\right)_{t t}= & \rho_{h}^{-1} f_{2, s s}+\left\langle\frac{M+P_{\hat{s}}}{\rho}\right\rangle f_{0, s s s s}+\frac{1}{2}\left\langle L^{2}\right\rangle\left(\phi_{0, s}{ }^{2} \cos \phi_{0}\right)_{t t} \\
\left(\phi_{2} \cos \phi_{0}\right)_{t t}= & \rho_{h}^{-1} g_{2, s s}+\left\langle\frac{M+P_{\hat{s}}}{\rho}\right\rangle g_{0, s s s s}+\frac{1}{2}\left\langle L^{2}\right\rangle\left(\phi_{0, s}{ }^{2} \sin \phi_{0}\right)_{t t} \\
\rho_{h} \phi_{2, t t}= & E_{h} \phi_{2, s s}-c^{2}\langle\rho\rangle \phi_{2}\left(1-\cos \phi_{0}\right) \\
& -f_{2} \sin \phi_{0}+g_{2} \cos \phi_{0}-\frac{c^{2} \rho_{h}\left\langle L^{2}\right\rangle}{2} \phi_{0, s}{ }^{2} \sin \phi_{0} \\
& +\left\langle E\left(N+Q_{\hat{s}}\right)\right\rangle \phi_{0, s s s s}-\langle\rho N\rangle \phi_{0, s s t t} \\
& +\left\langle E H_{K, \hat{s}}\right\rangle \chi_{s}+\left\langle E H_{L, \hat{s}}\right\rangle \lambda_{s}
\end{aligned}
$$

This is a system for $\left(f_{2}, g_{2}, \phi_{2}\right)$, which we solve using a traveling wave reduction. Integrating twice the reduced first two equations with zero boundary conditions at infinity yields:

$$
\begin{aligned}
& f_{2}=-c^{2} \rho_{h} \phi_{2} \sin \phi_{0}-\rho_{h}\left\langle\frac{M+P_{\hat{s}}}{\rho}\right\rangle f_{0, \xi \xi}-\frac{c^{2}}{2} \rho_{h}\left\langle L^{2}\right\rangle \phi_{0, s}{ }^{2} \cos \phi_{0} \\
& g_{2}=c^{2} \rho_{h} \phi_{2} \cos \phi_{0}-\rho_{h}\left\langle\frac{M+P_{\hat{s}}}{\rho}\right\rangle g_{0, \xi \xi}-\frac{c^{2}}{2} \rho_{h}\left\langle L^{2}\right\rangle \phi_{0, s}{ }^{2} \sin \phi_{0}
\end{aligned}
$$


Eliminating $f_{2}$ and $g_{2}$ from the reduced equation (73c) yields the following ODE for $\phi_{2}(\xi)$ :

$$
\phi_{2, \xi \xi}-\frac{1}{\ell^{2}} \phi_{2} \cos \phi_{0}=\psi(\xi),
$$

where $\ell$ is given by (37), and $\psi$ is

$$
\begin{aligned}
\psi(\xi)=\frac{1}{\ell^{2}}\left(\frac{\left\langle E\left(N+Q_{\hat{s}}\right)\right\rangle}{c^{2} \rho_{h}}-\frac{\langle\rho N\rangle}{\rho_{h}}\right) \phi_{0, \xi \xi \xi \xi} & \\
+\frac{1}{\ell^{2}}\left(\left\langle E H_{K, \hat{s}}\right\rangle-\left\langle E H_{L, \hat{s}}\right\rangle\right. & \left.\left(1-\cos \phi_{0}\right)-\rho_{h}\left\langle\frac{M+P_{\hat{s}}}{\rho}\right\rangle\right) \phi_{0, \xi \xi} \\
& -\frac{1}{\ell^{2}}\left(\left\langle E H_{L, \hat{s}}\right\rangle+\frac{\left\langle L^{2}\right\rangle}{2}\right) \phi_{0, \xi}{ }^{2} \sin \phi_{0}
\end{aligned}
$$

The homogeneous part of equation (75) is the same as the equation for $\phi_{1}$ (56). One solution (satisfying the boundary conditions for $\phi_{1}$ ) was found to be (57):

$$
\phi_{2, h o m}^{(1)}=\phi_{0, \xi} .
$$

The other solution, found by a reduction $\phi_{2}(\xi)=u(\xi) \phi_{0, \xi}(\xi)$ of the homogeneous equation, is:

$$
\phi_{2, h o m}^{(2)}=\phi_{0, \xi} \int \frac{1}{\phi_{0, \xi}^{2}(\xi)} \mathrm{d} \xi .
$$

A particular solution of the equation (75) is now obtained by variation of parameters

$$
\phi_{2, \text { part }}=\phi_{2, \text { hom }}^{(1)} \int \frac{W_{1}(x)}{W(x)} \mathrm{d} x+\phi_{2, h o m}^{(2)} \int \frac{W_{2}(x)}{W(x)} \mathrm{d} x,
$$

where $W$ is the Wronskian determinant for the homogeneous basis (77), (78), and

$$
W_{1}(\xi):=-\psi(\xi) \phi_{2, h o m}^{(2)}, \quad W_{2}(\xi):=\psi(\xi) \phi_{2, h o m}^{(1)} .
$$

The explicit form of the particular solution $\phi_{2}$ is far too complex to be reproduced here, but it is found to satisfy null boundary conditions at infinity.

\section{Homogenized traveling wave solution}

Using a homogenization approach, we have obtained a leading order solution $\phi_{0}$ and the first correction $\phi_{1}$ in terms of the angle variable,

$$
\begin{aligned}
& \phi_{0}(\xi)=4 \arctan \mathrm{e}^{\frac{\xi-\xi_{0}}{\ell}}, \\
& \phi_{1}(\xi)=\phi_{0, \xi}(\xi)=\frac{2}{\ell} \operatorname{sech} \frac{\xi-\xi_{0}}{\ell},
\end{aligned}
$$

as well as the second correction $\phi_{2}$, given by (79). Whereas $\phi_{0}$ and $\phi_{1}$ depend on the material parameters, the tension $T$, and the wave speed $c$ only through 
the characteristic length $\ell$ (37), the second-order correction explicitly depends on all material parameters $E_{a}, E_{b}, \rho_{a}, \rho_{b}, \alpha$, and the wave speed $c$ (note the dependence of $\psi$ on various averaged quantities in (76), each being an expression involving the material parameters).

The graphs of the three solutions are shown in Figures 2, 3, and 4 . The

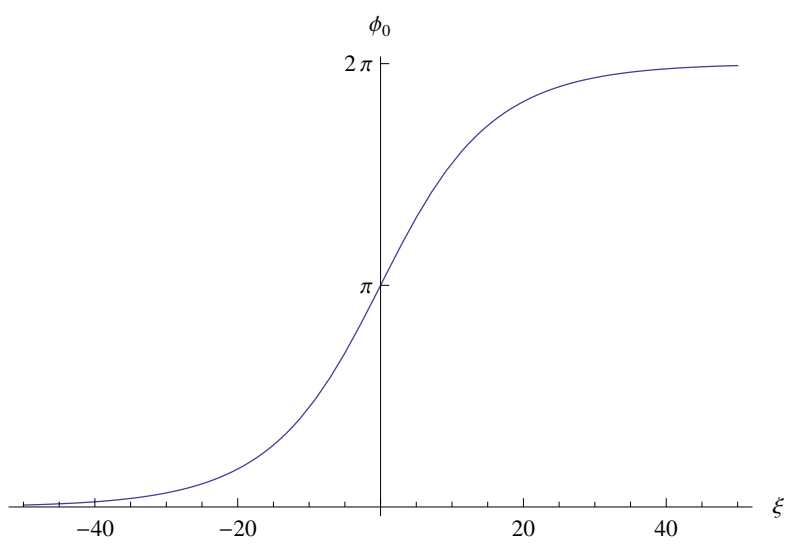

Figure 2: Homogeneous rod solution (39) for the angle. $\left(\xi_{0}=0, \ell=10\right)$

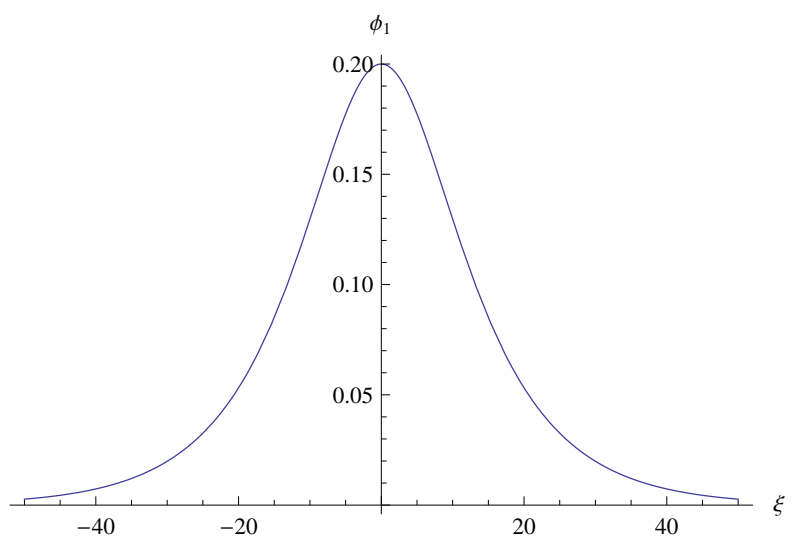

Figure 3: First order correction (57) in terms of the angle. $\left(\xi_{0}=0, \ell=10\right)$

effect of the first correction is to increase the angle $\phi$ within a localized region, which coincides with the extent of the loop (compare with Figure 2).

In order to view the solution in terms of the shape of the rod in the $(x, y)$ plane, rather than integrating the $x$ and $y$ equations (1d) and (1e) for the combined angle $\phi=\phi_{0}+\epsilon \phi_{1}+\epsilon^{2} \phi_{2}$, we carry out the same multiple scale expansion as seen above for these two equations. 


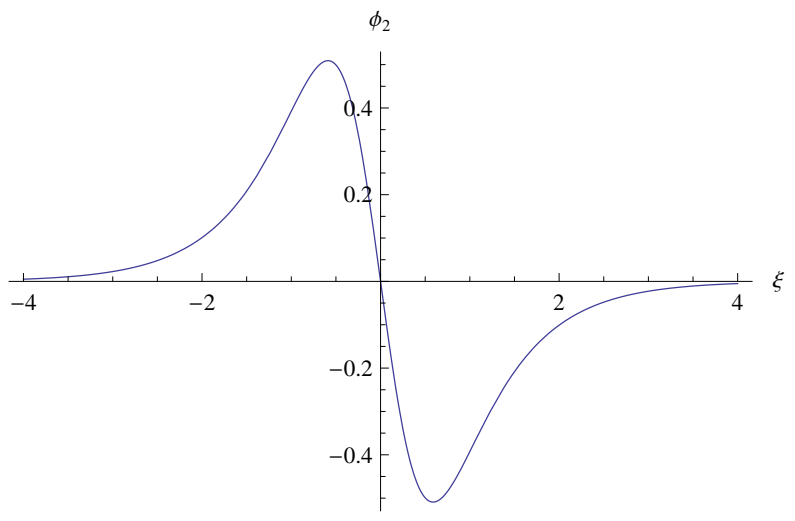

Figure 4: Numeric solution for the second order correction (79) in terms of the angle. $\left(\xi_{0}=0, \ell=10, E_{1}=1, E_{2}=\frac{1}{2}, \rho_{1}=0.8, \rho_{2}=1, \alpha=0.2, T=1\right.$, and the wave speed $c$ is given by (37))

\subsection{Leading order solution}

Collected terms of order $O\left(\epsilon^{0}\right)$ for the Cartesian coordinates system and averaging over the unit cell yields:

$$
\begin{aligned}
& x_{0, s}=\cos \phi_{0}, \\
& y_{0, s}=\sin \phi_{0} .
\end{aligned}
$$

The solution of the zero-order system (81) is the well-known loop solution on a homogeneous rod, depicted in Figure 5.

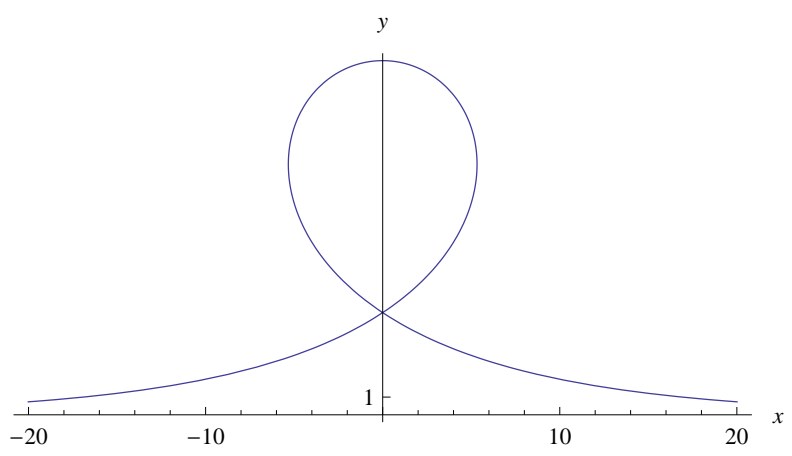

Figure 5: Loop-like traveling wave solution for the homogenous rod, corresponding to the solution (39) for the angle. $\left(\xi_{0}=0, \ell=10\right)$ 


\subsection{First-order correction}

Collecting terms of order $O(\epsilon)$ and applying the averaging operator yields:

$$
\begin{aligned}
& x_{1, s}=-\phi_{1} \sin \phi_{0}, \\
& y_{1, s}=\phi_{1} \cos \phi_{0},
\end{aligned}
$$

This gives the following solution in terms of the Cartesian coordinates:

$$
\begin{aligned}
& x_{1}(\xi)=\cos \left(4 \arctan \mathrm{e}^{\frac{\xi-\xi_{0}}{\ell}}\right)-1, \\
& y_{1}(\xi)=\sin \left(4 \arctan \mathrm{e}^{\frac{\xi-\xi_{0}}{\ell}}\right),
\end{aligned}
$$

where the integration constants have been set by null Dirichlet boundary conditions at infinity. The graphs of the two coordinate solutions are shown in Figure 6 . The effect of the first correction in the $x$ direction is to move the homogeneous solution loop to the left (see Figure 6a). In the $y$ direction, the "front part" of the loop, i.e. the part corresponding to values of the independent variable $\xi<\xi_{0}$, experiences a shift upwards, while the $\xi>\xi_{0}$ part shifts downwards (see Figure 6b). This effect is illustrated in Figure 7, where relatively large values of $\epsilon$ have been used in order to accentuate the effect. The first-order correction conserves the arc length of the loop, since $\lim _{\xi \rightarrow \infty} x_{1}(\xi)=\lim _{\xi \rightarrow-\infty} x_{1}(\xi)$.

\subsection{Second-order correction}

The $O\left(\epsilon^{2}\right)$ system yields:

$$
\begin{aligned}
& x_{2, s}=-\frac{1}{2} \phi_{1}^{2} \cos \phi_{0}-\phi_{2} \sin \phi_{0}, \\
& y_{2, s}=-\frac{1}{2} \phi_{1}^{2} \sin \phi_{0}+\phi_{2} \cos \phi_{0},
\end{aligned}
$$

While the leading-order and first-order systems are solved analytically above, the solutions we obtained for the the second-order system are numerical. Integrating the coordinates $x_{2}$ and $y_{2}$ from (84) yields solutions depicted in Figures $8 \mathrm{a}$ and $8 \mathrm{~b}$ The arc length of the loop is altered by the second correction: note the shift in the $x$-coordinate versus the arc length variable $\xi$ in Figure $8 \mathrm{a}$. In the horizontal direction, the loop gets stretched out (cf. Figure 8a), while in the vertical direction, the tails get slightly pushed upwards, while the central

part of the loop is significantly pulled downwards (cf. Figure 8b). The effect of the second correction on the overall shape of the loop is shown in Figure 9 for different values of $\epsilon$ (compare with corresponding values of $\epsilon$ in Figure 7 ).

\section{Conclusion}

We have examined planar loop traveling wave solutions on a heterogeneous, inextensible and unshearable elastic rod using a multiple scales homogenization 


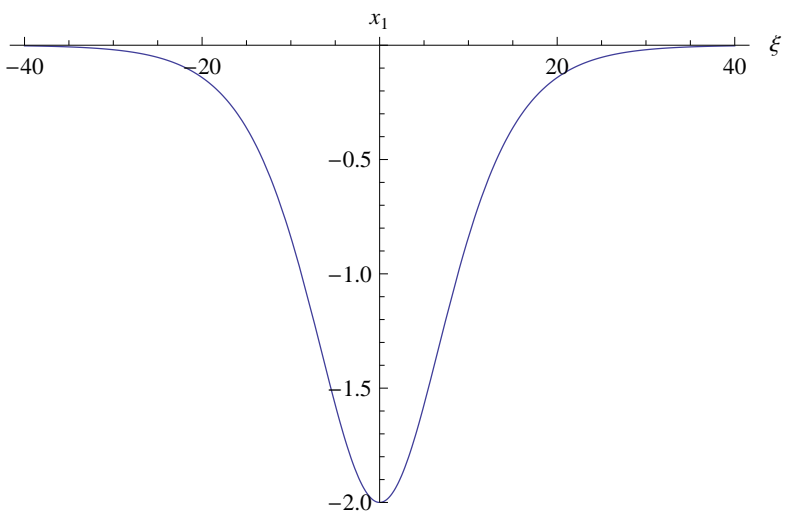

(a) $x_{1}$

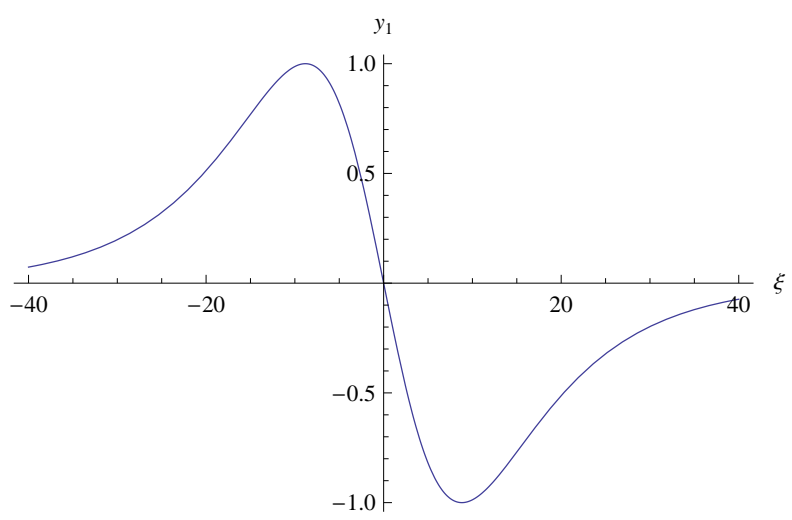

(b) $y_{1}$

Figure 6: First correction traveling wave solution (83). $\left(\xi_{0}=0, \ell=10\right)$

technique, where the heterogeneity consisted in a periodic microstructure of two different material properties.

The leading order balance (30) is a system describing a homogeneous rod, and allowed us to identify the effective material properties. In the case of the density $\rho$, it is just the bulk density $\rho_{h}=\langle\rho\rangle$, but as far as the Young modulus is concerned, the effective value is found to be $E_{h}=\left\langle E^{-1}\right\rangle^{-1}$.

Two lowest-order corrections have been found and shown to distort the homogeneous-type loop, breaking its symmetry. The effect of the corrections is shown in Figure 9. From the effect of the second-order boundary conditions, we can infer that an initially travelling loop on a rod with periodic micro-structure would eventually deform and loose its travelling wave structure as expected due to dispersion effects.

Acknowledgments: This publication is based in part upon work supported by 


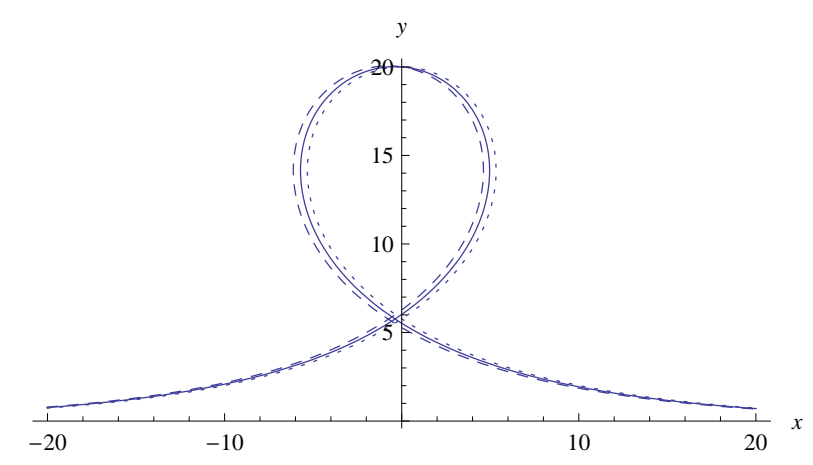

Figure 7: Homogenized solution up to the first correction, $\phi=\phi_{0}+\epsilon \phi_{1}$, shown in the Cartesian plane for two different values of $\epsilon: \epsilon=\frac{3}{8}$ (solid curve), and $\epsilon=\frac{3}{4}$ (dashed curve). The homogeneous solution $(\epsilon=0)$ is shown in dotted. $\left(\xi_{0}=0, \ell=10\right)$

the National Science Foundation under grant DMS-0907773 and by Award No. KUK-C1-013-04, made by King Abdullah University of Science and Technology (KAUST) (AG). AG is a Wolfson/Royal Society Merit Award holder.

\section{References}

[1] Michel Nizette and Alain Goriely. Towards a classification of Euler-Kirchhoff filaments. J. Math. Phys., 40(6):2830-2866, June 1999.

[2] Bojan Đuričković, Alain Goriely, and Giuseppe Saccomandi. Compact waves on planar elastic rods. International Journal of Non-Linear Mechanics, 44 (5):538-544, June 2009. doi: 10.1016/j.ijnonlinmec.2008.10.007.

[3] Jacob Fish and Wen Chen. Higher-order homogenization of initial/boundary-value problem. J. Engrg. Mech., 127(12):1223-1230, December 2001. doi: 10.1061/(ASCE)0733-9399(2001)127:12(1223).

[4] Tyler McMillen and Alain Goriely. Whip waves. Phys. D, 184:192-225, 2003. doi: 10.1016/S0167-2789(03)00221-5.

[5] J. Kevorkian and J.D. Cole. Multiple scale and singular perturbation methods. Springer, 1996.

[6] W. Chen and J. Fish. A dispersive model for wave propagation in periodic heterogeneous media based on homogenization with multiple spatial and temporal scales. J. Appl. Mech., 68(2):153, March 2001. doi: $10.1115 / 1.1357165$. 


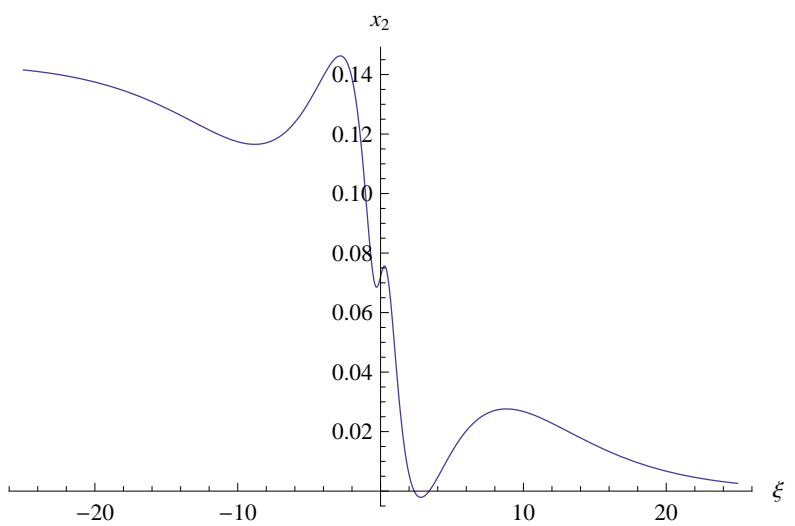

(a) $x_{2}$

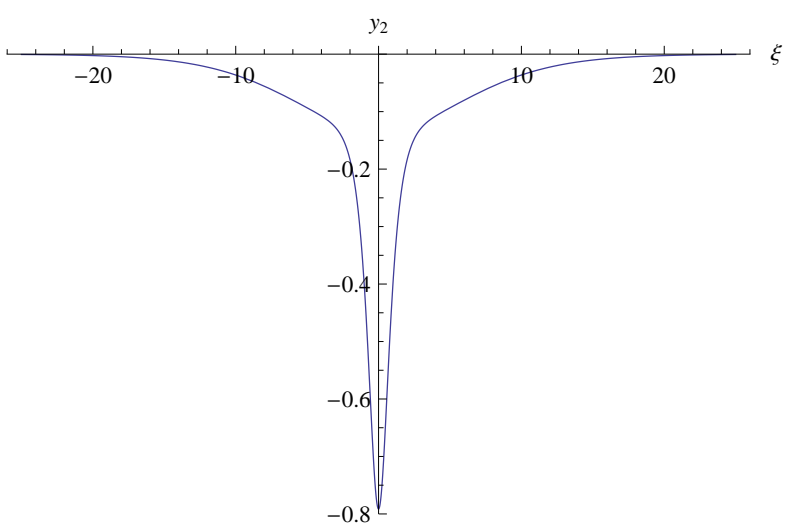

(b) $y_{2}$

Figure 8: Second correction to the traveling wave solution for the Cartesian coordinates, (84) $\left(\xi_{0}=0, \ell=10, E_{1}=1, E_{2}=\frac{1}{2}, \rho_{1}=0.8, \rho_{2}=1, \alpha=0.2\right.$, $T=1$, and the wave speed $c$ is given by (37)) 


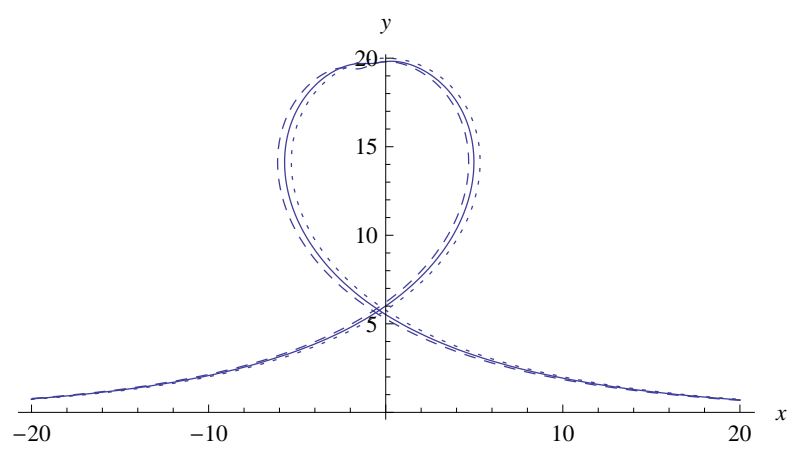

Figure 9: Homogenized solution up to the second correction, $\phi=\phi_{0}+\epsilon \phi_{1}+\epsilon^{2} \phi_{2}$, shown in the Cartesian plane for two different values of $\epsilon: \epsilon=\frac{3}{8}$ (solid curve), and $\epsilon=\frac{3}{4}$ (dashed curve). The homogeneous solution $(\epsilon=0)$ is shown in dotted. $\left(\xi_{0}=0, \ell=10, E_{1}=1, E_{2}=\frac{1}{2}, \rho_{1}=0.8, \rho_{2}=1, \alpha=0.2, T=1\right.$, and the wave speed $c$ is given by (37)) 



\section{RECENT REPORTS}

02/11 Self-diffusion in remodelling and growth Epstein

Goriely

03/11 Spontaneous rotational inversion in Phycomyces Goriely

Tabor

04/11 From individual to collective behaviour of coupled velocity jump Erban processes: a locust example

Haskovec

05/11 Solving Eigenvalue problems on curved surfaces using the closest MacDonald point method

Brandman

Ruuth

06/11 A numerical methodology for the Painleve equations

Fornberg

Weideman

07/11 Strong stability preserving two-step Runke-Kutta methods

Ketcheson

Gotlieb

MacDonald

08/11 Hysteresis and Post Walrasian Economics

Cross

McNamara

Kalachev

Pokrovskii

09/11 A locally adaptive time-stepping algorithm for petroleum reservoir

McNamara simulations

Bowen

Dellar

10/11 On the predictions and limitations of the BeckerDoring model for reaction kinetics in micellar surfactant solutions

Griffiths

Bain

Breward

Colegate

Howell

Waters

11/11 Dynamics of the Tear Film

Braun

12/11 The infuence of receptor-mediated interactions on reactiondiffusion mechanisms of cellular self-organisation

Klikaa

Baker

Headon

Gaffney

13/11 Quasi-steady state analysis of two-dimensional random intermittent search processes

Bressloff

Newby

14/11 A Constrained Approach to Multiscale Stochastic Simulation of Chemically Reacting Systems

Cotter

Zygalakis

Kevrekidis

Erban

15/11 The Two Regime Method for optimizing stochastic reactiondiffusion simulations

Flegg

Chapman

Erban

Kirchartz

Pieters

Kirkpatrick 
18/11 Asymptotic analysis of a pile-up of edge dislocation Hall

19/11 A perturbation analysis of spontaneous action potential initiation Keener1 by stochastic ion channels Newby

20/11 Hybrid modelling of individual movement and collective behaviour Franz

Erban

21/11 A novel model for one-dimensional morphoelasticity. Part I: The- Hall oretical foundations Menon

McCue

McElwain

22/11 A novel model for one-dimensional morphoelasticity. Part II: Ap- Hall plication to the contraction of fibroblast-populated collagen lat- Menon tices

$\mathrm{McCue}$

McElwain

23/11 Positive or negative Poynting efect? The role of adscititious in-

Mihai equalities in hyperelastic materials

Goriely

$\mathrm{McCue}$

McElwain

24/11 On approaches to modelling lattice dislocations Hall

Markenscoff

Copies of these, and any other OCCAM reports can be obtained from:

Oxford Centre for Collaborative Applied Mathematics

Mathematical Institute

24 - 29 St Giles'

Oxford

OX1 3LB

England

www.maths.ox.ac.uk/occam 